\title{
Paraffin induced sclerosing lipogranuloma of eyelids and anterior orbit following endonasal sinus surgery
}

\author{
Heinrich Witschel, Kathrin Geiger
}

\begin{abstract}
During the past 5 years we have seen 10 patients who had developed sclerosing lipogranuloma of the lid and orbit after endonasal sinus surgery with subsequent ipsilateral monocular haematoma. The histopathological examination of all and the additional nuclear magnetic resonance spectroscopic examination of four surgical specimens in combination with the typical clinical course led to the conclusion that the granulomas were caused by small paraffin droplets. These in turn stemmed from the ointment plugs applied to the sinuses at the end of the operation, and were washed out into the surrounding tissues by the postoperative haemorrhage. As the chronic and recurring granulomas can lead to considerable cosmetic and functional impairment, the application of ointment plugs at the end of sinus surgery should be abandoned, especially when peri- or postoperative bleeding occurs. (Br F Ophthalmol 1994; 78: 61-65)
\end{abstract}

Paraffin-induced sclerosing lipogranuloma has long been known as a typical complication after intradermal injection of paraffin in dermatology or cosmetic surgery. ${ }^{1-4} \mathrm{~A}$ few cases have been reported in which sclerosing lipogranuloma of the lids was caused by paraffin-containing ointment plugs of the lacrimal drainage system, ${ }^{5-7}$ by topical ointment application to the conjunctiva, ${ }^{8}$ or by hydraulic oil injury to the lid skin. 9 Though Brusis $^{10}$ in 1984 reported a case of granulation tumour of the orbit after ointment tamponade of the maxillary sinus, no attention has as yet been drawn to the fact that paraffin granuloma may be a typical and severe complication of endonasal sinus surgery. Strempel," in a paper about orbital lipogranulomas, described three patients who had undergone sinus surgery and had developed sclerosing lipogranuloma. The author related this reactive inflammation to a nonspecific surgical trauma, but was not aware of paraffin as the possible causal agent.

During the past few years we have encountered a number of patients who developed typical sclerosing lipogranuloma or paraffin granuloma several weeks or months after endonasal sinus surgery. This retrospective study was undertaken to work out the typical clinical course and the histopathological picture, to clarify the aetiology and the pathogenesis, and to help prevent future cases. Two patients, who impressively demonstrated the diagnostic difficulties in this disease, will be described in more detail.

\section{Material and methods}

Between 1987 and 1991 the ophthalmopathological laboratory of the University Eye Hospital in Freiburg received surgical specimens of 10 patients with sclerosing lipogranuloma of the lids and the anterior orbit which had developed after endonasal sinus surgery. The clinical data, including those from the ear, nose, and throat clinics were taken from the patients' records. In most cases a computed tomogram was available.

The surgical specimens were fixed in $10 \%$ buffered formalin and embedded in paraffin wax. The following staining methods were applied: haematoxylin and eosin, periodic acid Schiff, trichrome Goldner, and Berlin blue for iron. Frozen tissue of some specimens was stained for neutral fats with Sudan red.

Formalin fixed tissue of the last four patients was extracted with cold chloroform and examined by nuclear magnetic resonance spectroscopy. The spectra of ${ }^{1} \mathrm{H}$ and ${ }^{13} \mathrm{C}$ were taken for differentiation and were compared with those of healthy lid tissue from other patients.

\section{Results}

CLINICAL COURSE

The important clinical data of the 10 patients are summarised in Table 1. All patients were operated on for polypoidal sinusitis by endonasal surgery. In all cases bleeding occurred during surgery and bilateral or monolateral monocular haematoma developed. In nine patients a tamponade of the operated sinus with paraffin-containing ointment plugs was documented; in one patient it can only be suspected.

After the haemorrhage had subsided, the patients first realised a slowly growing irregular tumour of the lids 3 to 4 weeks after the endonasal operation, sometimes in connection with an inflammatory reddening of the covering skin. In one patient the lid tumour became
$9 \quad 59, M$ Monocular haematoma left side $\begin{gathered}\text { restriction of upward gaze } \\ \text { Swelling of left eyelids } 6 \text { months }\end{gathered}$ 10 50, F Right monocular haematoma Swelling of right eyelids 12 months 


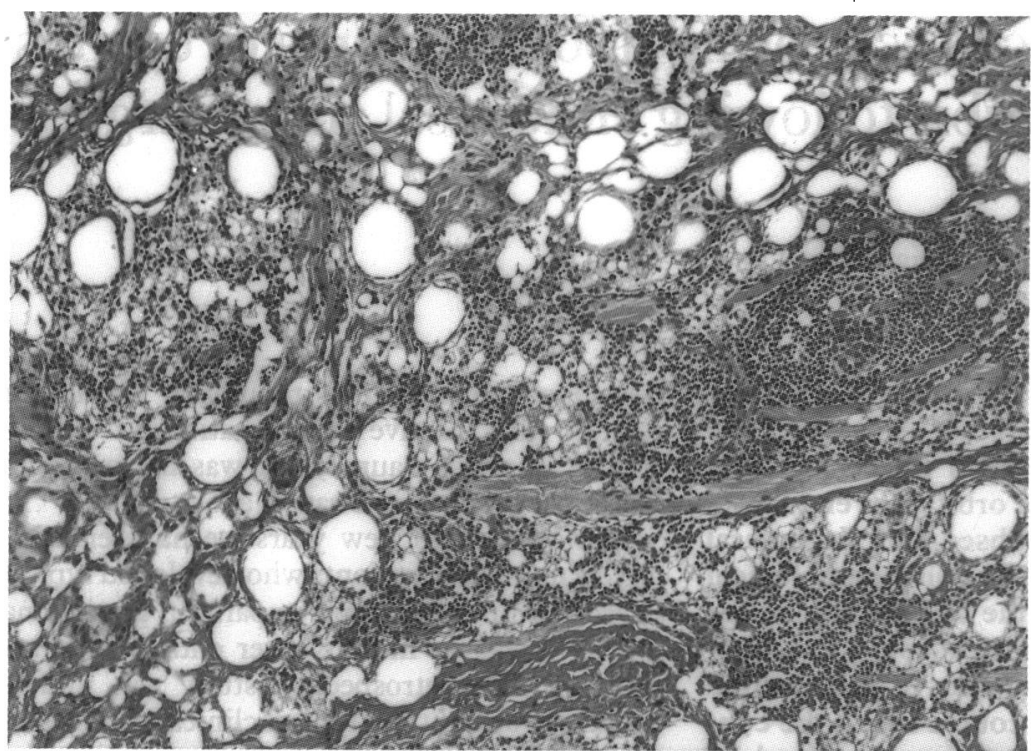

Figure 1 Sclerosing lipogranuloma (paraffinoma) of the lid 14 months after ear, nose, and throat surgery. 'Swiss cheese' appearance with multiple vacuoles surrounded by epithelioid and giant celts, foam cells, lymphocytes, and remnants of the orbicularis muscle. Haematoxylin and eosin, $\times 60$.

evident only 1 year and in another patient only 2 years postoperatively. Functional impairment, like ptosis of the upper lid or restricted motility of the eye occurred in four patients. Computed tomography, which was available in eight patients, showed a diffuse infiltration of the lid tissue sometimes including the anterior orbit.

On operation of the lid tumours, which was carried out 3 months to 4 years after endonasal surgery, the infiltrating nature of the lesions also became evident. The granulomas were never encapsulated and had grown into the orbicularis muscle in all and into the anterior orbit in three cases. A complete surgical resection was not possible in any patient.

Postoperative follow up ranged from 3 months to 4 years. Only one patient seemed to be completely cured clinically. In all the others either the symptoms persisted or the tumour recurred. Three patients were treated postoperatively with steroids and two of them responded well.

\section{HISTOPATHOLOGY}

All specimens showed the typical histological picture of a paraffin granuloma. The normal lid structures were replaced by diffusely infiltrat-

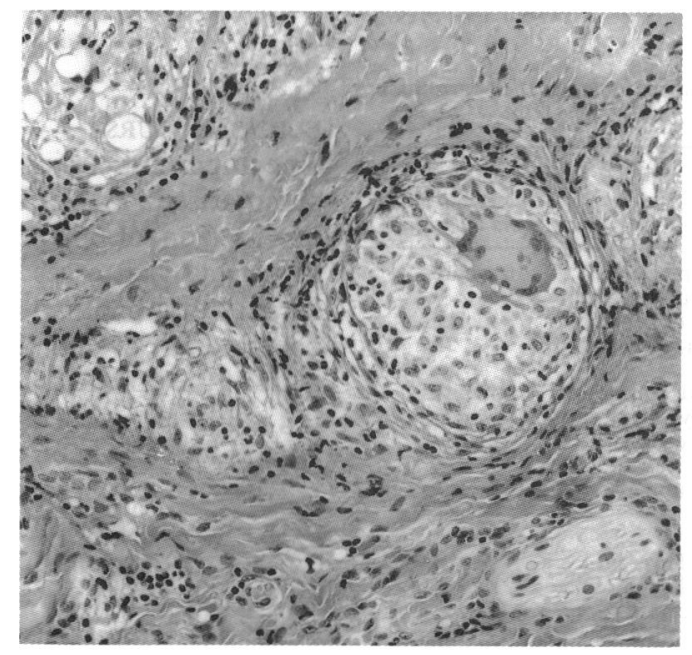

Fig 2A ing granulomatous tissue leaving only small remnants of the orbicularis muscle intact. In fresh lesions the granulomas consisted mainly of epithelioid cells, often with foamy cytoplasm, interspersed lymphocytes and occasional plasma cells, and many empty vacuoles giving the appearance of a 'swiss cheese pattern' (Fig 1). While the small vacuoles were found to be intracellular or to lie between adjacent cells, the larger vacuoles were surrounded by layers of elongated epithelioid cells, often forming multinucleated giant cells. In older lesions the single granulomas consisting of tightly packed epithelioid cells were separated by small strands of collagenous tissue containing vessels and lymph follicles, sometimes with germinal centres. The longer the granulomas existed the more the vacuoles decreased in number while strands of dense connective tissue predominated, surrounding small islands of epithelioid cells. Larger vacuoles were often replaced by concentric layers of hyaline tissue (Fig 2). The staining for neutral fat was faintly positive in some of the vacuoles, while staining for iron was always negative.

\section{NUCLEAR MAGNETIC RESONANCE SPECTROSCOPY}

In all four specimens examined high concentrations of n-alcanes, the major constituents of paraffin could be detected (Fig 3 ).

\section{Case reports}

CASE 1

This 35-year-old woman (No 6 in Table 1) appeared in our eye hospital in January 1989 with an irregular hard tumour of the right lower lid and the nasal canthal region (Fig 4), which had slowly grown during the last months. The patient had seen her local practitioner and her ophthalmologist, but a definite diagnosis could not be made. As the results of computed tomography were suspicious of an infiltrating neoplasm the patient was referred to our clinic for operation. An excisional biopsy was done and the histopathological diagnosis was 'sclerosing lipogranuloma' possibly due to paraffin or a similar substance (Fig 2). The patient denied having had any operation or injection more recently. On inquiry, however, she admitted that she had

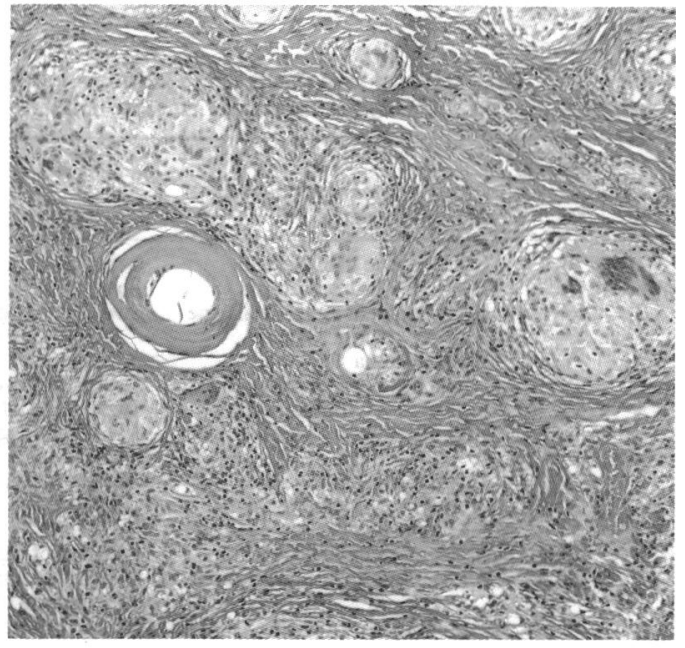

Fig $2 B$
Figure 2 Sclerosing (paraffinoma) of the lid 15 years after ear, nose, and epithelioid cells and occasional foreign body gian cells surrounded by dens vacuoles. Haematoxylin and eosin, $\times 100$. (B) Marked sclerosis with small epithelioid granulomas, few tiny vacuoles. A large 


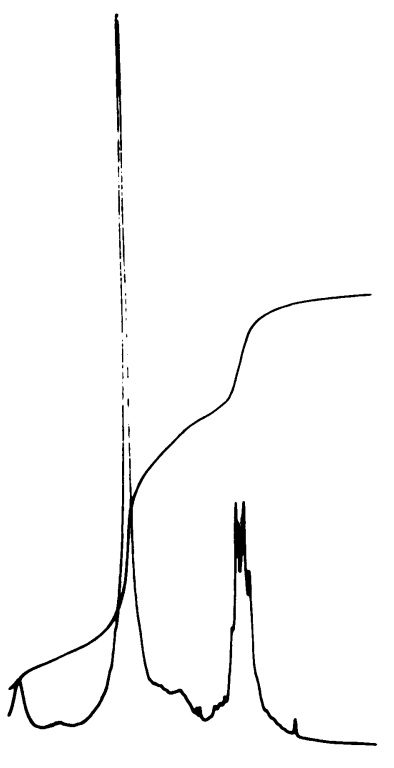

1.5
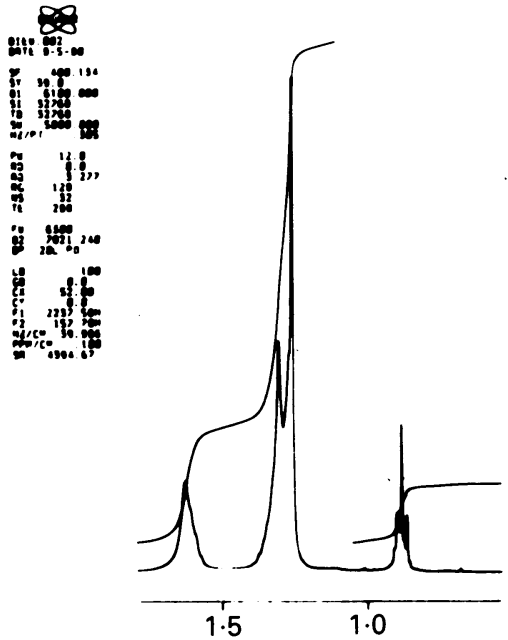

Figure 3 Nuclear magnetic resonance spectra of lid tissue from patient No 2 (left) and from paraffin as used in ointments (right), both showing high concentrations of n-alcanes.

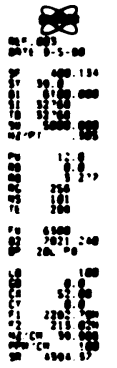

been operated on for a tumour in the same region 15 years earlier and that a slight swelling had remained ever since. We were able to retrieve her old records and the histological slides. While the microscopic picture was that of a typical fresh paraffin granuloma, the records revealed that the patient had undergone nasal sinus surgery with severe subsequent bleeding 3 months before the first lid operation. Thus the causal relationship between the ear, nose, and throat operation and the lid granuloma and its recurrence 15 years later could finally be established.

\section{CASE 2}

The 24-year-old man (No 8 in Table 1) was referred to our eye hospital in October 1991 because of an increasing swelling of the right upper lid with ptosis, downward displacement of the right globe, and partially restricted motility (Fig 5). One and a half year before, in April 1990, he had undergone right paranasal sinus surgery which had been followed by a massive monocular haematoma. When the lid was operated on, a diffusely infiltrating yellowish tumour was found which could not be completely excised. The histopathological examination of the excised tissue revealed a typical paraffin granuloma with densely packed epithelioid cells (Fig 6). Nuclear magnetic resonance spectroscopy demonstrated

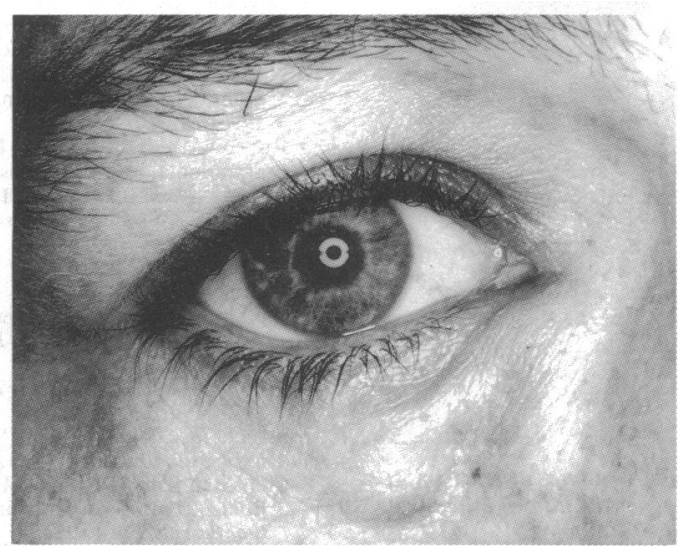

large amounts of n-alcanes, the typical constituents of paraffin. On request, the ear, nose, and throat surgeon confirmed that the paranasal sinuses had been plugged with an ointment tampon at the end of the endonasal surgery. Three months later the lid tumour recurred. As the patient was not willing to undergo a second operation without general anaesthesia, he was sent to his local practitioner for preoperative evaluation. On chest $x$ ray and computed tomography a swelling of the hilar lymph nodes and several round foci of the lungs were detected (Fig 7) and the patient was referred to a pulmonary clinic without our knowledge. There, a bronchoalveolar lavage and a bronchial biopsy were performed yielding lymphocytes and a small epithelioid granuloma in the bronchial mucosa, respectively. As, in addition, the angiotensin converting enzyme (ACE) level was elevated to $88 \mathrm{U} / \mathrm{ml}$ (normal $30 \mathrm{U} / \mathrm{ml}$ ), the diagnosis of sarcoidosis was made and the lid tumour was felt to be yet another manifestation of a sarcoid granuloma. The patient was put on systemic steroids for 2 months, and when he returned to our eye hospital the infiltration of the lid and the lungs had completely subsided.

The histological slides of the bronchial biopsy were re-examined in our laboratory and compared with the slides of the lid tumour. The type of granuloma was the same in both specimens and was more likely to be caused by paraffin than to be a sarcoid granuloma.

\section{Discussion}

The typical histological pattern, which is well known from paraffin granulomas of other sites, ${ }^{14}$ and the fact that in four of the 10 cases high concentrations of $\mathrm{n}$-alcanes could be demonstrated by nuclear magnetic resonance spectroscopy leave little doubt that paraffin was also the triggering agent in our patients. All our patients had undergone endonasal sinus surgery. In nine of the $\mathbf{1 0}$ there is proof that the sinuses were plugged at the end of the operation with ointment tampons containing paraffin as a major constituent. Thus, it is very likely that ear, nose, and throat surgery and the paraffin granulomas of lid and orbit are causally related. Since endonasal sinus surgery is a very common operation and postoperative paraffin granuloma, however, a rather rare event, there must be an additional factor for initiating the chronic disease. This factor appears to be postoperative haemorrhage. All our patients had a postoperative monocular haematoma and in all of them the paraffin granuloma developed at the same site after the haematoma had subsided. During endonasal sinus surgery an injury of the extremely thin bony wall between the sinuses and the orbit cannot always be avoided. ${ }^{12}$ If small branches of the ethmoidal or sphenopalatine arteries are severed considerable bleeding may occur, spreading to the anterior orbit and through the emissaries in the orbital septum and also to the lid or, in case of anteriorly located ethmoidal cells, directly to the lid. The blood emulsifies the ointment and distributes it to the surrounding tissues. After the blood has been absorbed tiny droplets of paraffin remain. As
Sclerosing lipogranuloma of the lids. Recurrence 15 years after the first lid operation following 3 months after ear nose, and throat surgery.

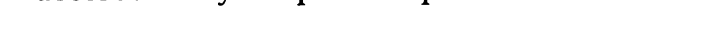




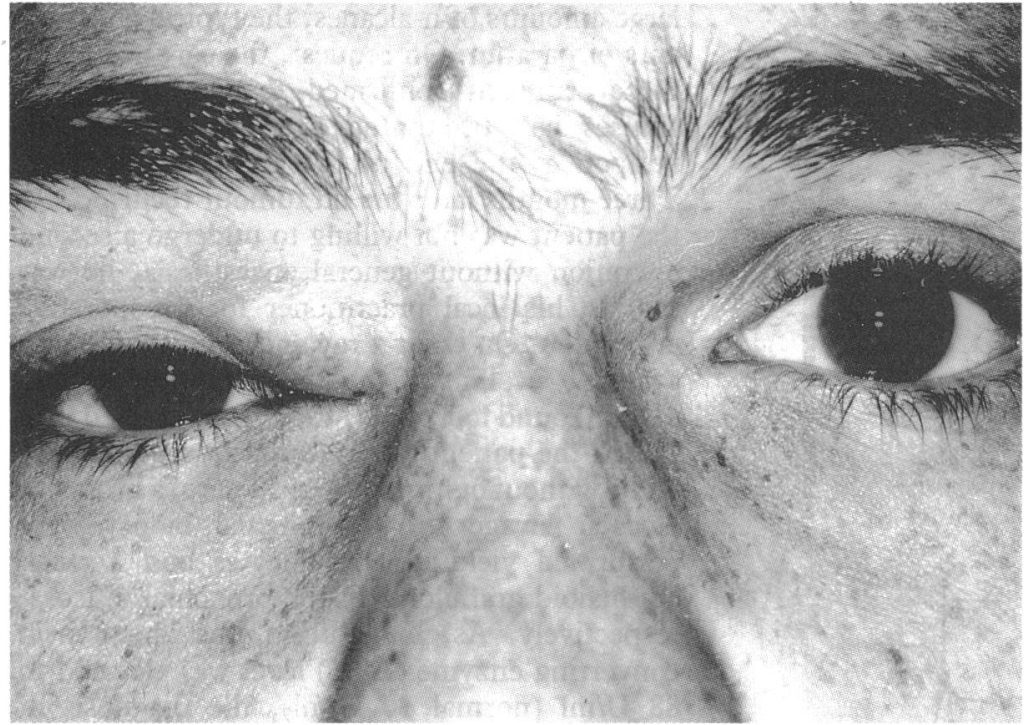

Figure 5 Case 2. Sclerosing lipogranuloma of the right upper and lower lid and the nasal canthal region 17 months after ear, nose, and throat surgery. Ptosis, slight downward displacement, and restricted motility of the right globe.

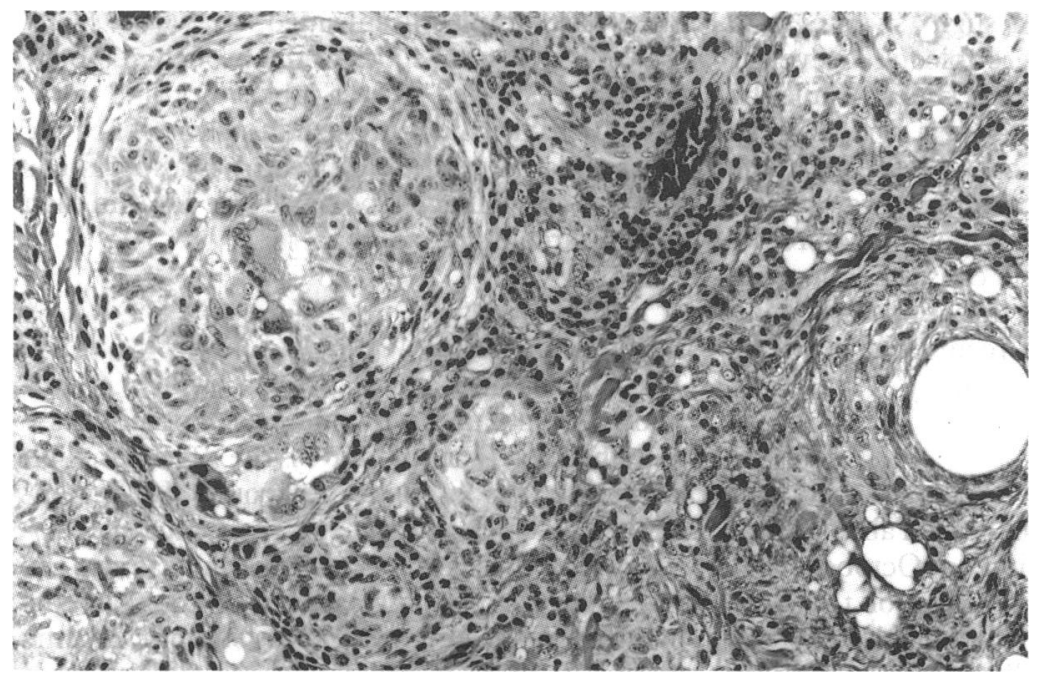

Figure 6 Case 2. Sclerosing lipogranuloma of the lids 17 months after ear, nose, and throat surgery. Epithelioid granulomas, foreign body giant cells, foam cells, lipid vacuoles, and lymphocytes. Haematoxylin and eosin, $\times 125$. they cannot be degraded by cellular enzymes they act as foreign bodies eliciting a diffuse chronic granulomatous inflammation.

Many ear, nose, and throat surgeons seem not to be aware of the fact that chronic paraffin granuloma can be a complication of sinus surgery, when bleeding occurs and ointment plugs are used. As a consequence the patients are not informed, and when they see the ophthalmologist several weeks or months after ear, nose, and throat surgery the causal relationship is often overlooked. Even after the operation of the lid and orbital tumours the correct diagnosis is often not made because the pathologist may not be familiar with the typical histological pattern of paraffin granuloma. This delay in diagnosis is well illustrated by the first of our two cases described in more detail.

The second case is especially interesting because a generalised disease led to considerable confusion. We can, of course, not exclude the possibility that our patient indeed had sarcoidosis as well, especially since paraffin may trigger the development of sarcoidosis or sarcoid-like disease in predisposed patients. On the other hand, it is well known that paraffin itself - for example, after long term application of paraffincontaining ointments to the nasal mucosa, or to a tracheostoma, or after accidental or occupational ingestion, may lead to pulmonary granulomas identical to those found in our patient. ${ }^{13-17}$ The small bronchial biopsy specimen showed a microscopic pattern compatible with that of the lid tumour. Although the remaining findings were thought to be diagnostic for sarcoidosis, as the detection of lymphocytes in the bronchoalveolar lavage and the raised ACE level are indeed nonspecific, we strongly believe that the pulmonary lesions in our patient were due to the ingestion of paraffin caused by the endonasal sinus surgery.

Though not a common event, postoperative paraffin granuloma is a severe complication leading to chronic and recurrent disease with considerable functional impairment. The use of paraffin-containing ointment in sinus surgery should therefore be abandoned, especially if peri- or postoperative bleeding occurs.

We thank Professor Fritz, Ciba Geigy Basle, and Professor Prinzbach, Institute of Chemistry of the University of Freiburg,

1 Boynton JR, Searl SS, Heimer JL, Miller EA. Eyelid oleogranulomas caused by petroleous jelly injection. Arch Ophthalmol 1988; 106: 550-1.

2 Newcomer VD, Graham JH, Schaffer RR. Sclerosing lipogranuloma resulting from exogenous lipids. Arch Dermatol 1956; 73: 361-72.

3 Rupec $M$, Treech $W$, Braun-Falco $O$. The paraffine granuloma. Clinical, histopathologic and histochemical study. Derm Wschr 1965; 151: 129-40.

4 Urbach F, Wine SS, Johnson WC, Davies RE. Generalized paraffinoma (sclerosing lipogranuloma). Arch Dermatol paraffinoma (scler.

5 Baumann HE. Recurring, infiltrative and destructive lipogranuloma of the lid following treatment of relative stenosis granuloma of the lid following treatment of relative stenosis of the lower

6 Lieb W. Paraffinoma of the eyelid. Klin Monatsbl Augenheilkd 1987; 190: 125-6.

7 Struck HG, Tost F. The 'lacrimal drainage system seal' indications and risks. Akt Augenheilk 1992; 17: 153-6

8 Moolchandani J, Kazim M, Farber M, Latowitz J. Eyelid lipoid granuloma following topical ointment application. Ophthalmol Plast Reconstr Surg 1990; 6: 133-5.

Figure 7 Case $2 . \mathrm{X}$ ray of the chest 20 months after ear, nose, and throat surgery, showing swelling of the hilar lymph nodes and several round foci of the lungs. 
9 Wolter JR, Nelson CC. Eyelid lipogranuloma after hydraulic oil injury. Ophthalmol Plast Reconstr Surg 1991; 7: 133-7.

10 Brusis T. Granulation tumour of the orbit after ointmenttreatment of the maxillary sinus. HNO $1984 ; 34: 511-4$.

11 Strempel I. Orbital lipogranuloma. Ophthalmologica (Basle) 1987; 195: 104-8.

12 Rauchfuss A. Complications in endonasal sinus surgery: surgical anatomy, pathomechanisms, clinical implication and surgical procedures. $H N O$ 1990; 38: 309-16.

13 Cornacchia DJ, Snyder CH, Dupont DC, Yaron NS. Exogenous lipoid pneumonia. $f$ Am Osteopath Assoc 1989; 89: 659-63.

14 Penes MC, Vallon JJ, Sabot JF, Vallon C. GC/MS detection of paraffines in a case of lipoid pneumonia following occupational exposure to oil spray. $\mathcal{F}$ Anal Toxicol 1990; 14: 372-4. 15 Pujol JL, Barneon G, Bousquet J, Michel FB, Godard P. Interstitial pulmonary disease induced by occupational exposure to paraffin. Chest 1990; 97: 234-6.

16 Reyes de la Rocha S, Cuningham JC, Fox E. Lipoid pneumonia secondary to baby oil aspiration: a case report pneumonia secondary to baby oil aspiration: a case report $74-80$.

17 Vonmoos S, Leuenberger P, Beer V, de Haller R. Pleuropulmonary infection caused by mycobacterium smegmatis. Case description and literature review. Schweiz Med Wschr 1986; 116: 1852-6.

\section{History of ophthalmology}

\section{India in the 1900s}

Quite a few ophthalmologists headed for India around the turn of the last century, just as junior doctors head for the Antipodes today (although in India the pay was worse, not better). $\mathrm{R} \mathrm{H}$ Elliott was the most famous and reported his more singular experiences at the Ophthalmology Congress in 1916.

The main point he made was that because his area was so large and the queues in his outpatient clinics very long (so what's new?), his patients would invariably have been treated at home first. This did not preclude a cure: squirting breast milk into sore eyes and rubbing the soles with oil to 'draw the heat from the head' rather amused him.

What really annoyed Elliot and his colleagues, was that patients with persistent symptoms were taken to the local eye expert, invariably an old woman whose reputation was proportional to her years, in spite of the fact that, as Elliot says, 'her ignorance was as colossal as her confidence'.

Very unfortunately, traditional remedies consisted of applying pastes of tamarind (very nasty stuff), lime juice, iron filings, and cow dung to the afflicted eyeball. While admitting that the specialists' intentions are 'as angelic as the results are devilish,' Elliot was truly appalled by the results.

One particular example upset him greatly. $\mathrm{He}$ described a tall, thin man walking to clinic with a long stick held out behind him, to which three small children clung. The history was that some weeks ago the eyes of all three had become inflamed, and pastes from the village specialist were applied with high hopes and with vigour. The man was obviously fond of his offspring. As a result, five eyes were hopelessly scarred and the sixth nearly so. Hence the stick.

The cosmetic results of this were also unfortunate. Mukerjee, out of pity for a 13 year old girl whose marriage value was diminished by her scarred cornea, experimented with contact glass painted in oils to match the other eye, and achieved an excellent result.
Nor was superstition confined to the lower classes. A highly educated man was advised to wash his eyes daily in his own urine, and nearly lost his sight as a result. Presumably Elliot said some harsh words before treating him and packing him off to the 1917 equivalent of a genitourinary clinic.

Worse still, deliberate corneal irritation was a popular form of malingering. One merely had to walk to an outside wall of one's house, scrape a little limewash off and drop it in the conjunctival sac, to get the day off with 'catarrh of the eye.' There was a real risk of blindness from overdoing this, yet the trick was extremely popular with the local police. Elliot put paid to this by explaining to his staff how the milkiness of the eye and the occasional lime particle.did tend to give the game away, and the police desisted, while frequently alluding to the 'devilish cunning' of the ophthalmic hospital.

Planning operating lists was also fraught with difficulty, since local astrologers had strong views on which hours were most favourable. If lists were late, patients would up and go to have their horoscope recast for the next favourable lunar phase, by which time they would sometimes be blind.

Knowledge of the frustrations of 'traditional treatment' might well have put practitioners off; indeed applications for Indian service declined sharply around this time. However, the obvious explanation was the pay: many Lancet correspondents were making stinging remarks about 'pecuniary remuneration,' and complained that as shooting and racing were getting beyond medics' financial reach, they were coming home.

FIONA ROMAN

Elliot RH. Some eccentricities of Indian ophthalmological practice. BrF Ophthalmol 1917; 1: 75-98.

IMS. Emoluments in the Indian medical service. Lancet 1911; 1 124.

Mukerjee SK. Use of contact glass for cosmetic purposes. $\mathrm{Br} \mathcal{F}$ Ophthalmol 1938; 22: 43-5. 